\title{
Immunophenotypic Comparison of Testicular Sclerosing Sertoli Cell Tumors and Sertoli Cell Tumors
}

\section{Not Otherwise Specified}

Hector Mesa ${ }^{a}$ MD, Chen Zhang ${ }^{b}$ MD, Juan C. Manivel ${ }^{a, c}$ MD, Thomas M. Ulbright ${ }^{b}$ MD.

Departments of Pathology and Laboratory Medicine, a. Veterans Administration Health Care System, Minneapolis, MN, b. Indiana University School of Medicine, Indianapolis, IN, c. University of Minnesota School of Medicine, Minneapolis, MN

Corresponding author: Hector Mesa, MD; Hector.Mesa@va.gov Minneapolis VA-Health Care System Office BB-104, One Veterans Drive, Minneapolis, 55417 MN Phone: 612-467-2502 Fax: 612-725-2079

Keywords: Testicular neoplasms; Sertoli cells; immunohistochemistry; PAX8 and PAX2; receptors, androgen; $\beta$-catenin

Short title: Immunophenotype of Sclerosing SCT \& SCT-NOS

The manuscript, or parts of it, have not been and will not be submitted elsewhere for publication. All authors have read and approved the manuscript. All authors acknowledge substantial participation and responsibility for this work. The authors do not have conflicts of interest to declare. There are no funding sources to disclose. The study was performed in compliance with institutional guidelines (Veterans Health Administration handbook 1200.05).

This is the author's manuscript of the article published in final edited form as:

Mesa, H., Zhang, C., Manivel, J. C., \& Ulbright, T. M. (2017). Immunophenotypic Comparison of Testicular Sclerosing Sertoli Cell Tumors and Sertoli Cell Tumors Not Otherwise Specified. Human Pathology. https://doi.org/10.1016/j.humpath.2017.08.022 


\begin{abstract}
Testicular Sertoli cell tumors (SCT) are rare and most fall into the category of SCT-not otherwise specified (SCT-NOS). Only a few additional types of SCT are recognized. Sclerosing SCT (S-SCT), originally described in 1991, comprises a small fraction of SCTs and was considered a specific entity until the 2016 revision of the World Health Organization classification of non-germ cell tumors, where it was classified as a morphologic variant of SCT-NOS. In a recent study, differences in expression of PAX2/PAX8, inhibin, androgen receptor and S100 protein between SCT-NOS and S-SCT were noted in a small number of cases. In this interinstitutional study, we compared the expression of these markers and $\beta$-catenin in 11 cases each of SCT-NOS and S-SCT to determine if differences exist that could justify keeping a separate classification of these neoplasms. PAX2/PAX8 cocktail was the only marker that was significantly overexpressed in S-SCT. Expression of androgen receptors was strong in S-SCT and variable in SCT-NOS, but did not reach statistical significance. Expression of $\beta$-catenin was common in both, while inhibin was infrequent. The available material was insufficient for a conclusive evaluation of S100 protein expression. Overall, our results support the inclusion of S-SCT as a morphologic variant of SCT-NOS. Expression of PAX2/PAX8 in S-SCT may reflect an overactive epithelial to mesenchymal transition as has been shown in experimental models of acute and chronic seminiferous tubular injury and might be related to the process generating the stroma in these tumors.
\end{abstract}




\section{Immunophenotypic Comparison of Testicular Sclerosing Sertoli Cell Tumors and Sertoli Cell Tumors Not Otherwise Specified}

\section{Introduction}

Testicular Sertoli cell tumors (SCT) are rare: the incidence of testicular neoplasms in the US is 6/100,000/year, and of these SCTs comprise only 1\% [1,2]. Although morphologically diverse, most SCTs fall into the category of SCT-not otherwise specified (SCT-NOS), most are benign, and only a few additional types of SCT have been recognized [3]. Sclerosing SCT (S-SCT), originally described by Zukerberg, Young and Scully in 1991 [4], comprises a small fraction of SCTs. The largest series to date was compiled by Kao and the senior author (T.M.U.) [5], and was considered a specific entity until the 2016 revision of the World Health Organization (WHO) classification of non-germ cell tumors, in which it was designated a morphologic variant of SCT-NOS because both tumors shared clinical similarities, mutations of the $\beta$-catenin (CTNNB1) gene, and differed only in the amount of associated non-neoplastic stroma $[2,6]$. However, in a recent comparative immunophenotypic study differences in expression of PAX2/PAX8 cocktail, inhibin, androgen receptor and S100 protein between SCT-NOS and S-SCT [7] were noted, although the number of examined cases ( 3 of each) was too small to draw definitive conclusions. In this interinstitutional study, we compared the expression of these markers and $\beta$-catenin in 22 SCTs, including 11 cases each of SCT-NOS and S-SCT, to determine if aside from morphologic differences immunophenotypic differences also exist that might justify the separate classification of these neoplasms.

\section{Material and Methods}

SCT-NOS and S-SCT from previous studies were retrieved from the pathology databases from Indiana University (Indianapolis, IN, USA) and hospitals affiliated with the University of Minnesota (Minneapolis, MN, USA). Given the rarity of these tumors and difficulty in obtaining study cases, the expression of 
PAX2/PAX8 cocktail, inhibin, androgen receptors, S100 protein and $\beta$-catenin was evaluated in already performed immunohistochemical stains, and, in cases with available unstained slides or paraffin embedded blocks, newly performed stains, prioritizing PAX2/PAX8 cocktail> inhibin> androgen receptors $>\beta$-catenin $>$ S100. Nodules of immature tubules (Sertoli cell nodules, Pick adenomas/tubular congeries), androgen insensitivity syndrome-associated lesions, and malignant SCTs were excluded. Immunohistochemical studies performed included inhibin A (Novacastra, catalog \# NCL-L-Inhibin A, Newcastle upon Tyne, UK; dilution 1:1500), androgen receptor (Bio SB, Santa Barbara, CA, catalog \# BSB 6074; dilution 1:10), PAX2 (Bio SB, Santa Barbara, CA, catalog \# BSB 2566, pre-dilute), PAX8 (Cell Marque, Rocklin, CA; catalog \# 363M-14, dilution:1:200), $\beta$-catenin (Cell Marque, Rocklin, CA; catalog \# 224M-14; dilution 1:300) and S100 protein (Novacastra, catalog \#CL-L-S100P, Newcastle upon Tyne, UK; dilution 1:1600). Immunohistochemistry was performed on a Leica BOND-III automated stainer (Leica Biosystems, Melbourne, Australia) using EDTA buffer antigen retrieval protocols. PAX2/PAX8 cocktail, $\beta$ catenin and androgen receptor were considered positive only if nuclear staining was present; inhibin and S100 protein was considered positive if cytoplasmic staining was present. The intensity of expression was graded as: $0=$ negative, $1=$ weak, $2=$ moderate, $3=$ strong relative to the internal control cells (if present) or external control cells in each preparation, and the area of expression as: $0=$ negative, $1=1-5 \%, 2=6-50 \%$ and $3=>50 \%$. Statistical analysis was performed using MedCalc Statistical Software version 16.8 (MedCalc Software bvba, Ostend, Belgium; https://www.medcalc.org; 2016). The study was performed in compliance with institutional guidelines (Veterans Health Administration handbook 1200.05). 


\section{Results}

A total of 22 cases, including 11 cases each of S-SCT and SCT-NOS, were retrieved, the majority from the consultation files of the senior author (T.M.U.). Table 1 summarizes the results of the immunohistochemical studies performed. Expression of PAX2/PAX8 cocktail was demonstrated in most S-SCT (8/10; 80\%), while most SCT-NOS were negative (1/11; 9\%). Expression of inhibin A and $\beta$-catenin was similar in both groups: most lesions were negative for inhibin A (S-SCT 2/9, 22\%; SCT-NOS 3/11, $27 \%$ ), but positive for $\beta$-catenin (S-SCT 8/10, 80\%; SCT-NOS 7/11, 64\%). Expression of androgen receptor was present in most tumors and overall it was the most consistent marker (S-SCT 8/9, 89\%; SCT-NOS 10/11, 91\%). In all S-SCT androgen receptor expression was strong, while in SCT-NOS it was variable: weak in 4/10, moderate in 4/10 and strong in 2/10. Expression of S100 protein was only tested in a few cases in each group: 3/11 S-SCT and 4/11 SCT-NOS; weak to moderate partial expression was present in all S-SCT, while all SCT-NOS were negative. A two-tailed t-test was calculated for all markers except S100 protein given the scant number of data points (table 1); the only marker that showed statistical significance was PAX2/PAX8 cocktail $(p=0.0006)$.

\section{Discussion}

The original description of S-SCT [4] in 1991 included 10 cases that comprised 5\% of testicular sex cordstromal cell tumors and $15 \%$ of SCT from the consultation and hospital files at the Massachusetts General Hospital (Boston, MA). These tumors diverged from SCT-NOS by their distinct morphology characterized by anastomosing tubules, nests or cords in a prominent hypocellular, collagenous background and scant entrapped Sertoli-cell-only tubules (fig 1). The clinical characteristics of the cohort did not differ from those of patients with SCT-NOS. One of the cases showed cytologic atypia, frequent mitoses, angioinvasion, and invaded the rete testis and epididymis; however, after a mean follow-up of $\sim 6$ years none had evidence of recurrence or metastasis after simple orchiectomy. The 
described histologic features were distinct enough that subsequent studies followed, mostly as case reports. The definition was later narrowed in the WHO and AFIP classifications to require that the fibrous stroma should comprise at least $50 \%$ of the tumor $[3,8]$ because focal fibrosis or deposition of basement membrane like collagen is common in SCT and Sertoli cell nodules, respectively. Segregation of this tumor was based both on its distinct morphology and the benign clinical course, in contrast to SCT-NOS. In the largest series of S-SCT compiled by Kao et al. in 2014, however, one of 20 cases pursued a malignant course and led to the patient's death [5]. The recognition of a malignant case showed that aside from the exaggerated non-neoplastic fibrous stroma there were no distinctive clinical and prognostic differences between S-SCT and SCT-NOS; the biologic behavior of individual tumors can be predicted more or less accurately by established clinicopathologic criteria for SCTs $[3,8]$.

Further evidence linking S-SCT and SCT-NOS was presented in 2014 when Perrone et al. found that CTNNB1 exon 3 mutations are common in SCT-NOS and S-SCT and correlate with nuclear expression/accumulation of $\beta$-catenin and cyclin D1 [9]. A subsequent study of a larger number of cases by 2 of the authors (C.Z. and T.U.M.) verified the frequent expression of nuclear $\beta$-catenin in tumors of both morphologic types [6]. The accumulated evidence, therefore, led to the discontinuation of S-SCT as a separate entity in the 2016 WHO classification, where it was considered a morphologic variant of SCTNOS. Nonetheless, a comparative immunophenotypic study of SCTs using an extensive panel in 2017 by two of the authors (H.M., C.M.) reported differences in the expression of PAX2/PAX8 cocktail, inhibin, androgen receptor and S100 protein between S-SCT and SCT-NOS; however, that study included only 3 cases of each category, making it impossible to perform any meaningful statistical analysis [7] (fig1). We have now performed an interinstitutional cooperative study to determine if the previous findings represented random phenotypic variations, or consistent immunophenotypic differences between these tumors that might justify maintaining S-SCT as a separate entity. Our results showed a highly significant statistical difference in the expression of PAX2/PAX8 cocktail, no differences in inhibin and $\beta$-catenin 
expression, and variation in the intensity of expression of androgen receptors that did not reach statistical significance. Expression of S100 protein was only documented in a few cases and could not be adequately evaluated. Despite the significant difference in expression of PAX2/PAX8 cocktail, overall SCT-NOS and S-SCT appear to have more commonalities than differences, supporting the recent modification of the WHO classification. In one of our cases the two tumors coexisted in the same testis, along with Sertoli cell nodules, similarly to one of the cases reported in the original series [4].

The capacity of inducing deposition of hypocellular fibrous stroma is inherent to non-neoplastic Sertoli cells and occurs frequently in the context of disease processes leading to testicular atrophy. We have previously shown consistent immunophenotypic differences between Sertoli cells from atrophic and non-atrophic tubules: gain of expression of pankeratin, calretinin, CD56 and nestin [7], suggesting that the fibrous obliteration of atrophic tubules represents an active process that involves activation of a mesenchymal/stromal physiologic alternative cell program under abnormal conditions. However, gain of expression of PAX2/PAX8 was not identified in atrophic tubules. It is apparent that this program is overactive in S-SCT, and may be related to the exaggerated fibrosis. We confirmed aberrant expression of PAX2/PAX8 cocktail in most S-SCT, a marker that in the testis has been traditionally associated with lesions derived from the rete testis and epididymis [10]. These proteins are involved in the regulation of gene expression: in the embryo, they cause repression of pluripotency genes and in the adult, participate in regulation of lineage specification. Interestingly, PAX2 and WT1 are required for epithelial to mesenchymal transition and are re-expressed in experimental models of tubular epithelial-to mesenchymal transition during acute and chronic injury [11]. Expression of this marker thus supports the argument that in S-SCT the "atrophic/injury"-program is overactive. We confirmed the consistent expression of $\beta$-catenin and androgen receptor, and the low frequency of expression of inhibin in both tumors; these results provide additional information regarding their use as ancillary tests in the work-up of testicular neoplasms. 


\section{Conclusions}

From the immunophenotypic differences previously noted in a limited sample of S-SCT and SCT-NOS only the expression of PAX2/PAX8 cocktail was statistically significant. Expression of $\beta$-catenin and androgen receptor is common in both tumors; expression of androgen receptors is strong in S-SCT and variable in SCT-NOS, however differences are not statistically significant. Expression of inhibin is uncommon in both tumors, and cannot be used as a reliable marker to support their diagnosis. Overall, our results confirm previous observations and support the inclusion of S-SCT as a morphologic variant of SCT-NOS. Strong sclerosis of the non-tumoral stroma could be related to activation of similar pathways to those leading to fibrous obliteration of seminiferous tubules in the context of atrophy or injury. 


\section{References}

1. Howlader N, Noone AM, Krapcho M, et al (eds). SEER Cancer Statistics Review, 1975-2014, National Cancer Institute. Bethesda, MD, https://seer.cancer.gov/csr/1975_2014/, based on November 2016 SEER data submission, posted to the SEER web site, April 2017.

2. Idrees MT, Ulbright TM, Oliva E, et al. The World Health Organization 2016 classification of testicular non-germ cell tumours: a review and update from the International Society of Urological Pathology Testis Consultation Panel. Histopathology. 2017; 70(4):513-521.

3. Ulbright TM, Young RH. AFIP Atlas of tumour pathology. Tumours of the testis and adjacent structures. Fourth series fascicle. Silver Spring, MD: ARP Press; 2013:271-86.

4. Zukerberg LR, Young RH, Scully RE. Sclerosing Sertoli cell tumor of the testis. A report of 10 cases. Am J Surg Pathol. 1991;15(9):829-34.

5. Kao C-S, Kum JB, Idrees, MT, Ulbright T. Sclerosing Sertoli Cell Tumor of the Testis: A Clinicopathologic Study of 20 Cases. Am J Surg Pathol. 2014;38(4):510-17.

6. Zhang C, Ulbright TM. Nuclear Localization of $\beta$-Catenin in Sertoli Cell Tumors and Other Sex Cord-Stromal Tumors of the Testis: An Immunohistochemical Study of 87 Cases. Am J Surg Pathol. 2015;39(10):1390-4.

7. Mesa, H., Gilles, S., Datta, M. W., Murugan, P., Larson, W., Dachel, S., \& Manivel, J. C. (2017). Comparative immunomorphology of testicular Sertoli and sertoliform tumors. Hum Pathol. 2017;61:181-189.

8. Moch H, Humphrey PA, Ulbright TM, Reuter VE (eds): World Health Organization Classification of Tumours of the Urinary System and Male Genital Organs (4th edition). IARC Press: Lyon 2016:228-232. 
9. Perrone F, Bertolotti A, Montemurro G, Paolini B, Pierotti MA, Colecchia M. Frequent mutation and nuclear localization of $\beta$-catenin in sertoli cell tumors of the testis. Am J Surg Pathol. $2014 ; 38(1): 66-71$.

10. Tong G-X, Memeo L, Colarossi C, Hamele-Bena D, et al. PAX8 and PAX2 Immunostaining Facilitates the Diagnosis of Primary Epithelial Neoplasms of the Male Genital Tract. Am J Surg Pathol. 2011;35:1473-83.

11. Blake JA, Ziman MR. Pax genes: regulators of lineage specification and progenitor cell maintenance. Development 2014;141:737-751. 


\section{Figure Legend}

Figure 1. Sclerosing Sertoli cell tumor. A. Low power showing anastomosing cords in a hypocellular fibrous background. The lower right corner shows a cluster of lipidized tumor cells (H\&E, 5X objective). B. Intermediate power showing alternation of cords and nests of cells with moderate and scant cytoplasm and dense fibrosis (H\&E, 20X objective). Immunohistochemical studies: C. Androgen receptor (nuclear): strong consistent expression (20X objective). D. Beta catenin (nuclear): strong consistent expression (20X objective). E. PAX2/PAX8 cocktail (nuclear): variable, moderate to strong expression (40X objective). F. S100 protein (cytoplasmic): variable, moderate to strong expression (20X objective). 
Table. Immunohistochemical stains

\begin{tabular}{|l|c|c|c|}
\hline \multicolumn{1}{|c|}{ Marker } & S-SCT SS \pm SD (n) & SCT-NOS SS \pm SD (n) & P value \\
\hline Pax2/Pax8 & $3.3 \pm 1.1(10)$ & $0.5 \pm 0.9(11)$ & $0.0006^{*}$ \\
\hline Inhibin A & $1.2 \pm 1.2(9)$ & $1.6 \pm 1.4(11)$ & 0.5071 \\
\hline Androgen Receptor & $5.3 \pm 1(9)$ & $4.4 \pm 1.1(11)$ & 0.0743 \\
\hline -Catenin & $4.6 \pm 1.3(10)$ & $3.8 \pm 1.5(11)$ & 0.800 \\
\hline S100 & $4.3 \pm 0.8(3)$ & $0(4)$ & N.C. \\
\hline
\end{tabular}

S-SCT=sclerosing Sertoli cell tumor. SCT-NOS= Sertoli Cell tumor not otherwise specified. SS= Staining Score: (mean intensity score + mean area score). Intensity score $=0$ : negative, 1 : weak, 2: moderate, 3: strong. Area score= 0: none, 1: 1-5\%, 2: 6-50\%, 3: $>51 \%$. SD= standard deviation. $\mathbf{n}=$ number of cases tested. $\mathbf{P}$ value $=$ calculated probability two-tailed T-Test. ${ }^{*}$ Significant $p$-value $<0.05$. N.C. $=$ not calculated due to insufficient data points. 

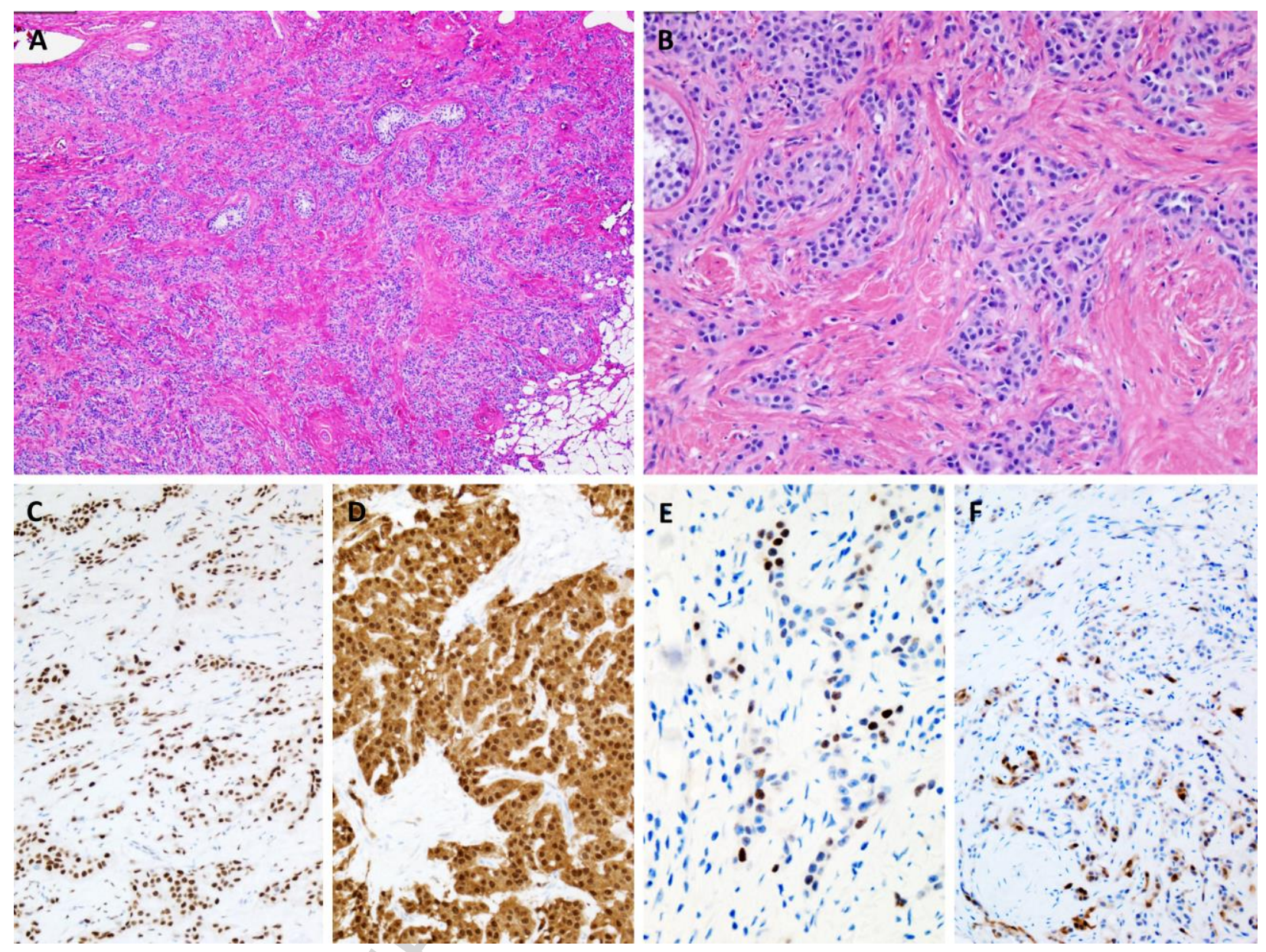

Figure 1 


\section{Highlights}

- Testicular Sertoli cell tumors (SCT) are rare and most are benign

- Most SCT are classified as not otherwise specified (SCT-NOS)

- $\quad$ Sclerosing SCT (S-SCT) is now considered a variant of SCT-NOS

- Immunophenotypic differences between SCT-NOS and SCT-NOS were reported

- $\quad$ S-SCT and SCT-NOS differ only in the amount of fibrosis and expression of PAX2/PAX8 